\title{
COMMUTATORS IN DIVISION RINGS
}

\section{BRUNO HARRIS}

1. Introduction. A theorem of Wedderburn [4] states: if every element of a finite dimensional associative algebra is a sum of nilpotent elements, then the algebra is nil. In [1], Kaplansky asked whether this theorem could be generalized to rings with minimum condition, and mentioned that an equivalent question is:

Does there exist a division ring $D$ with $D=[D, D]$ ? (Here $[D, D]$ denotes the subgroup of the additive group of $D$ generated by all additive commutators $[x, y]=x y-y x$.) An affirmative answer to the second question is equivalent to a negative answer to the first. In the finite dimensional case, the trace function shows that the second question has a negative answer.

In this paper we give an example of a division ring $D$ with $D=[D, D]$. In fact every element of $D$ is itself a commutator, and $D_{n}$ (the $n \times n$ matrix ring with coefficients in $D$ ) has the same property.

2. Construction of the division ring. Ore has shown that a noncommutative integral domain in which every two nonzero elements have a nonzero common right multiple can be imbedded in a division ring of (right) fractions [2]. He has also shown that the integral domain of differential polynomials in one variable with coefficients in a division ring has the common right multiple property. We show that a certain ring of differential polynomials in an infinite number of variables also has the common right multiple property, and that the division ring of fraction satisfies $D=[D, D]$ and even the stronger properties mentioned above.

Let $F$ be a field, $\left\{x_{i}\right\}_{i \in I}$ and $\left\{y_{i}\right\}_{i \in I}$ two infinite sets of variables with the same ordered index set $I$, and $P=F\left[\left\{x_{i}\right\},\left\{y_{i}\right\}\right]$ the set of formal polynomial expressions

$$
\sum a_{n\left(i_{1}\right) \cdots n\left(i_{k}\right) m\left(j_{1}\right) \cdots m\left(j_{l}\right)} x_{i_{1}}^{n\left(i_{1}\right)} \cdots x_{i_{k}}^{n\left(i_{k}\right)} y_{j_{1}}^{m\left(j_{1}\right)} \cdots y_{j_{l}}^{m\left(j_{l}\right)}
$$

where $i_{1}<\cdots<i_{k}, j_{1}<\cdots<j_{l}$ are in $I, a \cdots$ are in $F$, and only a finite number of terms occur in the sum. Define addition of polynomials the usual way, and multiplication by $\left[x_{i}, x_{j}\right]=0=\left[y_{i}, y_{j}\right]$, $\left[x_{i}, a\right]=0=\left[y_{i}, a\right]$ for $a \in F,\left[x_{i}, y_{i}\right]=1,\left[x_{i}, y_{j}\right]=0$ for $i \neq j$.

We show $P$ has the common right multiple property by a method due to Tamari [3]:

Received by the editors February 17, 1958. 
For $p \in P$, let deg $(p)$ denote the total degree of $p$ in the $x_{i}$ and $y_{i}$; then $\operatorname{deg}(p q)=\operatorname{deg} p+\operatorname{deg} q$. Let $p, q$ be nonzero polynomials, each of degree $<l$. The problem of finding a common nonzero right multiple $t=p r=q s$ of degree $l$ and such that $r$ and $s$ each contain only those variables that occur in $p$ or in $q$ is the same as the problem of solving a finite number of linear homogeneous equations in a finite number of unknowns: the coefficients of $r$ and $s$ are the unknowns, and each term of $t=p r=q s$ gives an equation. If $m$ is the larger of $\operatorname{deg} p, \operatorname{deg} q$ and $v$ is the number of variables that occur in $p$ or in $q$ then the number of equations is the same as the number of distinct monomials of degree $\leqq l$ in $v$ variables, i.e. $C_{v+l, v}$, and the number of terms in each of $r, s$ is $\geqq C_{v+l-m, v}$. Thus we have $C_{v+l, v}$ equations in at least $2 C_{v+l-m, v}$ unknowns. If $l \geqq m /\left(1-2^{-1 / v}\right)$, there are more unknowns than equations and a nonzero solution exists.

Let $D$ be the division ring of fractions $p / q=p q^{-1}, p, q \in P$. If $d=p q^{-1} \in D$, each of $p, q$ contains only a finite number of the variables $y_{i}$, and so, for some index $n, y_{n}$ does not occur in $p$ or in $q$. Then $\left[x_{n}, p\right]=0=\left[x_{n}, q\right]$ and $\left[x_{n}, d\right]=0,\left[x_{n}, y_{n} d\right]=\left[x_{n}, y_{n}\right] d=d$. Similarly, if $d_{1}, \cdots, d_{r}$ are a finite number of elements of $D$, then $y_{n}$ does not occur in any of the $d_{j}$ for some $n$, and so $\left[x_{n}, y_{n} d_{j}\right]=d_{j}, j=1, \cdots, r$. In particular if $\left(d_{i j}\right)$ is an $m \times m$ matrix over $D$, we can find $n$ such that $\left[x_{n}, y_{n} d_{i j}\right]=d_{i j}$, for all $i, j$. Let $c_{i j}=y_{n} d_{i j}$ and let $\left(x_{n}\right)$ be the matrix $x_{n} I, I$ the identity matrix; then $\left[\left(x_{n}\right),\left(c_{i j}\right)\right]=\left(d_{i j}\right)$.

3. Commutators and nilpotent matrices. We owe the first proposition and its proof to Professor Kaplansky, and the rest of the section is an amplification of his remarks in [1].

PROPOSITION 1. Let $R$ be any ring, $R_{n}$ the $n \times n$ matrix ring over $R$, ( $n \geqq 2)$. If $A \in R_{n}$ and trace of $A \in[R, R]$, then $A$ is a sum of nilpotent elements. In particular, if $A \in\left[R_{n}, R_{n}\right]$ then $A$ is a sum of nilpotent elements.

Proof. Any matrix is the sum of a diagonal matrix (i.e. one with zeros off the main diagonal) and two nilpotent matrices, so that for our purposes only the diagonal elements matter. The following $2 \times 2$ matrices are nilpotent:

$$
\left(\begin{array}{cc}
a b & a \\
-b a b & -b a
\end{array}\right) \text { and }\left(\begin{array}{rr}
-b a & -b a \\
b a & b a
\end{array}\right) \text {, also }\left(\begin{array}{rr}
-d & -d \\
d & d
\end{array}\right) \text {; }
$$

thus

$$
\left(\begin{array}{cc}
{[a b]} & * \\
* & 0
\end{array}\right)
$$


is a sum of nilpotent matrices.

Now let $A=\left(a_{i j}\right) \in R_{n}$, and $\sum_{1}^{n} a_{i i}=c \in[R, R]$, so $a_{11}=c$ $-\left(a_{22}+\cdots+a_{n n}\right)$. Let $x e_{i j}$ denote the matrix with $x$ in the $i, j$ position and zeros elsewhere. Using the above $2 \times 2$ matrices, we see that $c e_{11}$ and $-a_{i i} e_{11}+a_{i i} e_{i i}, i \geqq 2$, are sums of nilpotent matrices; therefore this also holds for $a_{11} e_{11}+\cdots+a_{n n} e_{n n}$, and finally for $A$. If $A \in\left[R_{n}, R_{n}\right]$, then trace of $A \in[R, R]$.

Proposition 2. Let $D$ be any division ring, $A$ an element of $D_{n}$, $n \geqq 2$. The following conditions are equivalent:

(a) $A \in\left[D_{n}, D_{n}\right]$,

(b) trace of $A \in[D, D]$,

(c) $A$ is a sum of nilpotent elements.

Proof. We have already shown $a \rightarrow b, b \rightarrow c$.

Define a "trace modulo commutators," $\operatorname{tr}(A)$, as the coset of the ordinary trace of $A$ in the factor group of the additive group of $D$ modulo $[D, D]$. Then $\operatorname{tr}(A+B)=\operatorname{tr}(A)+\operatorname{tr}(B)$, and $\operatorname{tr}(A B)$ $=\operatorname{tr}(B A)$. Now let $A$ be nilpotent: then there exists a nonsingular $B$ such that $B A B^{-1}$ is in Jordan canonical form, i.e. $B A B^{-1}$ is a sum of matrices of the form $n_{k, l}=e_{k+1, k}+e_{k+2, k+1}+\cdots+e_{l+1, l}, 1 \leqq k \leqq l$ $\leqq n-1$. Finally, $\left[\sum_{i=k}^{l+1}(i+1-k) e_{i, i}, n_{k, l}\right]=n_{k, l} ;$ thus $n_{k, l}$ and also $A$ are in $\left[R_{n}, R_{n}\right]$. This shows (c) $\rightarrow(\mathrm{a})$.

Corollary. If $D$ is a division ring such that $D=[D, D]$, then every element of $D_{n}, n \geqq 2$, is a sum of nilpotent elements.

\section{BIBLIOGRAPHY}

1. I. Kaplansky, Problems in the theory of rings, Report of a Conference on Linear Algebras, Long Island, New York, June, 1956, National Academy of Sciences. National Research Council Publication 502, pp. 1-3.

2. O. Ore, Linear equations in noncommutative fields, Ann. of Math. vol. 34 (1933) pp. $480-508$.

3. D. Tamari, On the embedding of Birkhoff-Witt rings in quotient fields, Proc. Amer. Math. Soc. vol. 4 (1953) pp. 197-202.

4. J. H. M. Wedderburn, Note on algebras, Ann. of Math. vol. 38 (1937) pp. 854856.

NORTHWESTERN UNIVERSITY 